\section{Neue Tabakpräventions-} kampagne: «lch bin stärker»

Im Jahr 2013 äusserten gemäss Suchtmonitoring Schweiz 57\% der täglich Rauchenden den Wunsch, mit dem Rauchen aufzuhören. Die neue Kampagne «Ich bin stärker» richtet sich in einer ersten Phase an diese Personen. Sie versucht, deren Motivation zu stärken, und macht sie auf bestehende Unterstützungsangebote aufmerksam. Aufhörwillige Raucherinnen und Raucher haben höhere Erfolgschancen, wenn sie professionelle Unterstützung in Anspruch nehmen, wie sie die Rauchstopplinie 0848000 181, Ärztinnen und Ärzte, Apotheken und beratende Fachstellen anbieten. Im Laufe der kommenden drei Jahre soll die Kampagne auch das positive Image einer rauchfreien Gesellschaft stärken. Von 2001 bis 2013 hat der Anteil der Rauchenden in der Schweizer Bevölkerung von $33 \%$ auf $25 \%$ abgenommen.

(BAG)

\section{Wirkstoff gegen tödlichen Muskelschwund}

Die Duchenne-Muskeldystrophie ist eine Erbkrankheit, die zum Muskelverlust und im Teenageralter zum Tod führt. Die genetische

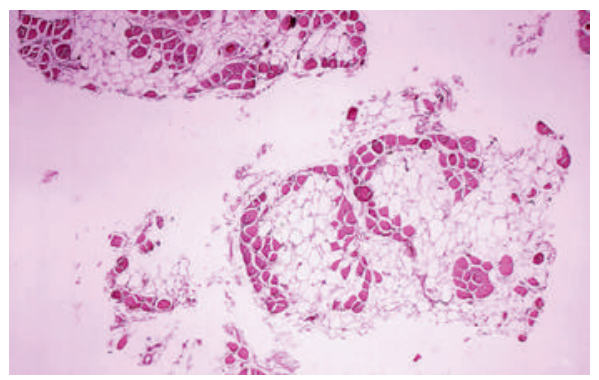

Histopathologisches Bild des Wadenmuskels eines Patienten mit Duchenne-Muskeldystrophie. Die Muskelfasern (rot) sind ausgedehnt durch Fettzellen (hell) ersetzt.

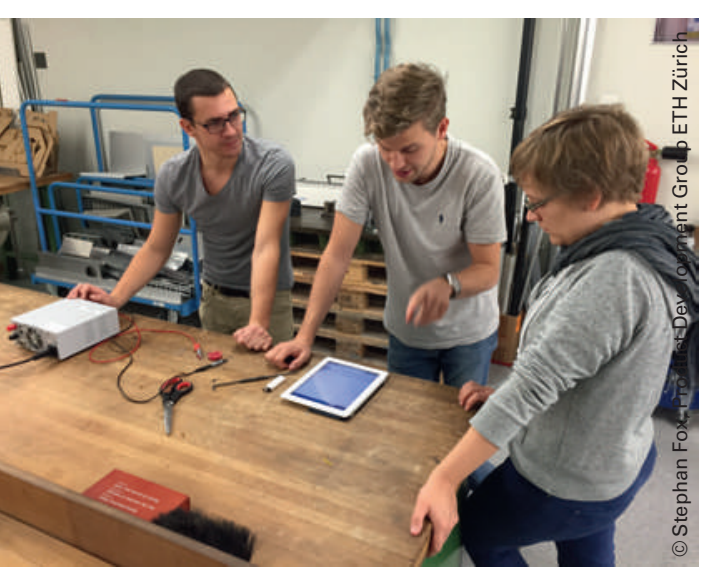

Studierende arbeiten an einem Schnelltest zur Erkennung von Streptokokken.

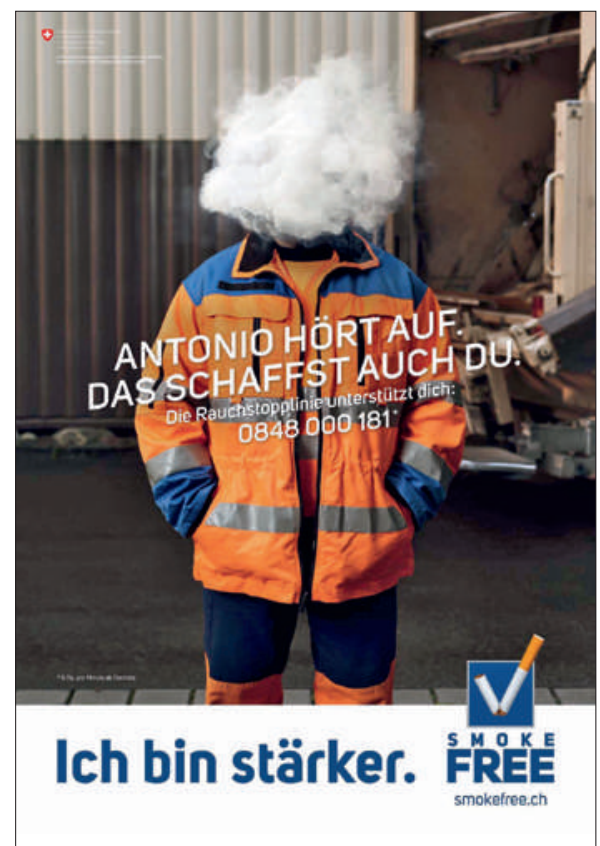

\section{Alcool et cancer: un lien encore méconnu}

La Journée mondiale contre le cancer du 4 février a donné l'occasion de rappeler que la consommation d'alcool est une cause de cancer méconnue. En Suisse, presque 30\% de tous les décès liés à l'alcool sont à mettre au compte de différents cancers. Ce sont avant tout les personnes de plus de 55 ans qui sont touchées par des cancers liés à l'alcool. Et selon la Ligue suisse contre le cancer, on estime que 4 à $8 \%$ de toutes les maladies cancéreuses sont liées à l'alcool. Cela signifie que sur 37000 maladies cancéreuses par an en Suisse, environ 1500 à 3000 sont imputables à la consommation d'alcool. Plus les quantités d'alcool augmentent, plus le risque de cancer augmente lui aussi, et cela dès un verre d'alcool par jour. Il y a plus de cas de décès liés à l'alcool dus au cancer qu'à la cirrhose du foie.

(Addiction Suisse)

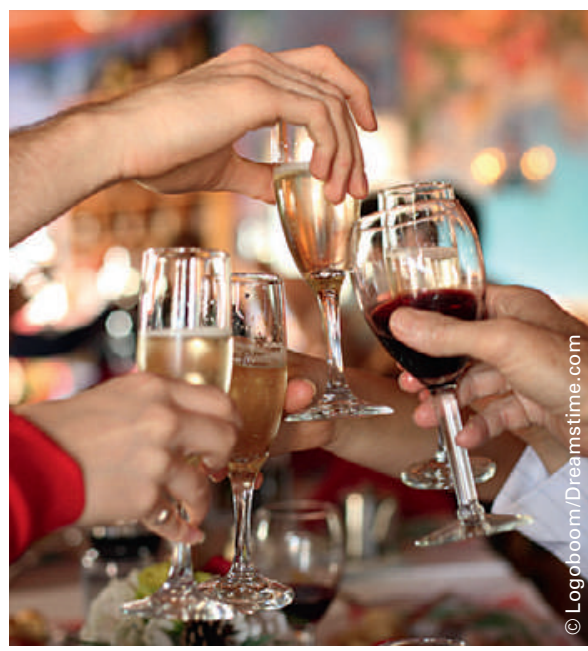

Trop méconnu: presque $30 \%$ des décès liés à I'alcool sont à mettre au compte de différents

(Universität Bern)

Ursache der Krankheit, die nur Knaben betrifft, liegt in einem Defekt in dem Gen, das für die Herstellung von Dystrophin verantwortlich ist. Dystrophin ist ein für die Muskelfunktion wichtiges Eiweiss. Als Folge der genetischen Mutationen wird entweder kein Dystrophin produziert oder lediglich eine funktionsuntüchtige Variante. Mit den bisher getesteten Wirkstoffen ist es noch nicht gelungen, einen signifikanten Heilungseffekt bei den Patienten zu erzielen, weil die entsprechenden Wirkstoffe noch zu wenig aktiv sind und vitale Muskeln wie das Herz gar nicht erreichen. Berner Forschende haben nun einen neuen Wirkstoff entwickelt und ihn zusammen mit einem internationalen Team erfolgreich getestet. cancers

\section{Gemeinsam tüfteln}

Wenn ein Kind mit Halsschmerzen, Schluckbeschwerden und einem entzündeten Rachen zum Kinderarzt geht, kann es sein, dass es unter einer "Streptokokken-Angina» leidet. Diese Bakterien in einem Test nachzuweisen, dauert in der Regel ein bis zwei Tage. Hilfreich wäre da ein zuverlässiger Schnelltest, den Ärztinnen und Ärzte in ihrer Praxis anwenden können. Einen solchen Test gibt es bis heute nicht. Seit vergangenem Herbstsemester erarbeiten Studierende der Medizin und der Maschineningenieurwissenschaften in einer gemeinsamen Lehrveranstaltung von Universität Zürich und ETH Zürich konzeptionelle Lösungsansätze, wie sich ein solcher Schnelltest realisieren liesse.

\section{La Semaine du Cerveau}

L'édition 2015 de la Semaine du Cerveau aura lieu du 16 au 21 mars dans différentes villes de Suisse. Cette année par exemple le CHUV de Lausanne propose, en partenariat avec le Pôle de recherche national "Synapsy», une édition résolument tournée vers l'avenir. Au menu, de nouvelles pistes thérapeutiques issues de la recherche et explorées par les praticiens: la contribution que le Human Brain Project apportera aux traitements de demain, ou encore les incroyables capacités d'adaptation du cerveau qui doit composer avec un œil bionique ou une main robotisée. L'autisme, surtout chez les jeunes, sera aussi au cœur d'un des forums. 